RAFAEL PEDEZZI

\title{
Prospecção de Enzimas no Fungo Purpureocillium lilacinum Utilizando as Técnicas de Proteoma: Produção Recombinante de Enzimas com Potencial Biotecnológico e Avaliação de Antifúngicos
}

Tese de Doutorado apresentada ao Programa de Pós-Graduação em Ciências Farmacêuticas da Faculdade de Ciências Farmacêuticas de Ribeirão Preto/USP para obtenção do Título de Doutor em Ciências

Área de concentração: Medicamentos e Cosméticos

Orientador: Prof. Dr. Hamilton Cabral

Ribeirão Preto 


\section{Resumo}

PEDEZZI, Rafael. Prospecção de Enzimas no Fungo Purpureocillium lilacinum Utilizando as Técnicas de Proteoma: Produção Recombinante de Enzimas com Potencial Biotecnológico e Avaliação de Antifúngicos2019. 115 f. Tese (Doutorado em Ciências) - Faculdade de Ciências Farmacêuticas de Ribeirão Preto, Universidade de São Paulo, RibeirãoPreto, 2019.

O fungo ascomiceto Purpureocillium lilacinum pode ser encontrado em solo, vegetação em decomposição, insetos, nematoides e outros locais. Apresenta grande importância agrícola, visto que pode ser utilizado como controle biológico por ser capaz de infectar e matar nematoides pragas de culturas, graças às suas enzimas, como peptidases e quitinases. Além disso, o fungo apresenta importância médica, pois é capaz de gerar infecção em humanos. A terapêutica das doenças infecciosas causadas por $P$. lilacinum é dificultada pelo fato de ele apresentar resistência a antifúngicos "azólicos", principais moléculas aplicadas na clínica médica atualmente. O presente trabalho teve como objetivo explorar molecularmente o fungo $P$. lilacinum com relação à sua capacidade de resistir a antifúngicos e à sua capacidade de produzir peptidases robustas, com potencial de aplicação biotecnológica. Ferramentas moleculares como transcriptômica e proteômica foram aplicadas para acessar os dados moleculares do fungo e viabilizar análises de expressão diferencial para verificar as respostas aos antifúngicos fluconazol e itraconazol e anotação gênica para prospecção de peptidases. Foi observado que $P$. lilacinum promove um aumento de expressão dos genes alvo dos antifúngicos e dos genes que codificam bombas de efluxo. Com esse mecanismo, não há acúmulo de antifúngico intracelular e há compensação numérica da enzima que está sendo inibida pelo antifúngico com mais expressão da mesma. Dessa forma, o fungo consegue manter a síntese de ergosterol normalizada. As enzimas prospectadas e expressas de maneira recombinante em Pichia pastoris foram uma serino peptidase ( $r P I$ SerPep) e uma metalo peptidase ( $r P l \_$MetPep). A rPI_SerPep se mostrou alcalina, com temperatura ótima aparente de $60^{\circ} \mathrm{C}$ e termoestável. Já a rPI_MetPep se mostrou ativa em pH neutro e com temperatura ótima aparente de $40{ }^{\circ} \mathrm{C}$. Essas enzimas se mostraram promissoras para futuros testes de aplicação biotecnológica.

Palavras chave Purpureocillium lilacinum, Resistência a antifúngicos, Peptidases fúngicas, Expressão recombinante, Transcriptoma 


\section{Introdução geral}

O fungo Purpureocillium lilacinum é um ascomiceto, está definido na classe dos Sordariomicetos, ordem Hypocreales e família dos Ophicordyciptaceos. Essas classe e família são compostas por fungos caracterizados por serem patógenos de plantas e insetos. Até 2011, P. lilacinum recebia o nome de Paecilomyces lilacinus. Seu nome foi alterado por ele apresentar discrepâncias com as características do gênero Paecilimyces, representada por fungos termoestáveis, por exemplo (Luangsa-ard et al., 2011). O fungo se caracterizou por apresentar conídios violeta ao iniciar sua esporulação em cultivos em placas de Petri e esse foi um dos motivos para moverem ele para 0 gênero Purpureocillium (Luangsa-ard et al., 2011). Apesar dessa mudança de nomes ter ocorrido em 2011, ainda há publicações de artigos se referindo ao nome Paecilomyces lilacinus (Burhan e Annon, 2019; Espadale et al., 2018 e Sanadhya et al., 2018).

Figura 1: Cultivo de P. lilacinum em placas de Petri. Cultura cultivada por 14 dias em meio de cultivo contendo extrato de malte. Pode-se observar coloração violeta, típica de esporulação do fungo

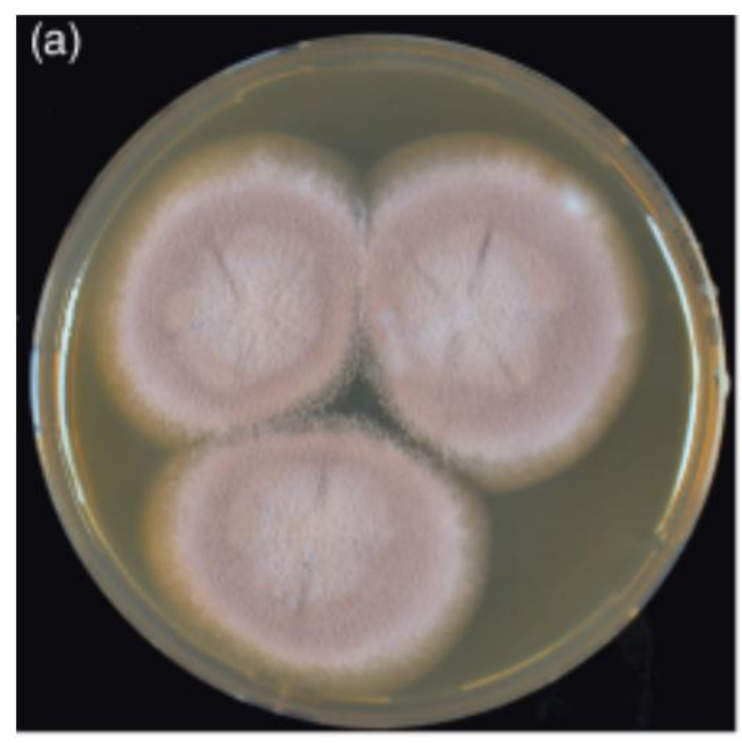

Fonte: Luangsa-ard et al., 2011

$P$. lilacinum é um fungo saprófito, encontrado em muitas localizações, mais frequentemente em solo, vegetação em decomposição, insetos e nematoides (Luangsaard et al., 2011). Existem relatos, inclusive, de associação de $P$. lilacinum com águasvivas (Yue et al., 2015). Em cultura, apresenta crescimento ótimo entre 26 e $30{ }^{\circ} \mathrm{C}$. 
Cresce em uma faixa de $\mathrm{pH}$ abrangente e tolera uma vasta diversidade de substratos (Banu et al., 2006 e Sun et al., 2006). Sua grande capacidade de adaptação aos mais diversos ambientes o torna um fungo com um grande potencial genético a ser explorado. Possui dois projetos genoma completos depositados no banco de dados Esembl (https://fungi.ensembl.org/index.html), mas, diante de seu potencial, ainda possui poucos recursos bem explorados.

Uma característica bem estudada de $P$. lilacinum é sua capacidade de infectar invertebrados, como insetos e nematoides. Por essa característica, o fungo vem sendo explorado como possível controle biológico de pragas, principalmente para os nematoides (Wang et al., 2016 e Xie et al., 2016). Após os primeiros relatos de infecção nos nematoides Meloidogyne incognita acrita e Globodera pallida, que atacam culturas de batatas, estudos posteriores foram realizados (Jatala et al., 1979 e Stirling et al., 1991). Verificou-se que a virulência se dá pela produção e secreção de enzimas e metabólitos secundários capazes de desestabilizar as membranas dos ovos dos nematoides (Wang et al., 2016, Wang et al., 2010). Dentre as enzimas produzidas estão peptidases e quitinases (Khan et al., 2004). Um metabólito secundário foi isolado do fungo por atuar nos mecanismos de infecção e foi denominado leucinostatina (Wang et al.,2016). Hoje já existe uma linhagem de P. lilacinum (PL251) registrada nos Estados Unidos como controle biológico de nematoides (Kiewnick et al., 2011). O estudo da aplicação deste organismo e seus recursos genéticos na biotecnologia vem ganhando força e se mostra muito promissor.

$P$. lilacinum também é observado infectando seres humanos. Os relatos mostram que não só pessoas imunossuprimidas podem ser infectadas, mas também pessoas com sistema imunológico saudável (Carey et al., 2003 e Pastor e Guarro, 2006). O maior número de relatos é o de oculomicoses, seguidos de infecções cutâneas e subcutâneas (Pastor e Guarro, 2006). Além disso, há outras infecções relatadas, como sinusite, perfuração do septo nasal, osteomielite, onicomicose, fungemia, endocardite, doença pulmonar cavitária e vaginite (Pastor e Guarro, 2006; Carey et al., 2003; Khalique et al., 2018; Shivaprasad et al., 2012; Khan et al., 2012; Raghavan et al., 2018 e Evans et al., 2016). 
Existe uma associação muito forte entre a causa dessas doenças e a utilização de materiais sintéticos contaminados. No caso das oculomicoses, por exemplo, os principais agentes inoculantes de fungo foram lentes implantadas, lentes de contato ou soluções de armazenamento de lentes contaminadas com P. lilacinum (Pastor e Guarro, 2006). Existem relatos de contaminação de material cirúrgico ou equipamento de tatuagem, que geraram infecções (Trinh e Angarone, 2017 e Khalique et al., 2018). Essa associação mostra que, mesmo com protocolos de esterilização e utilização de materiais sintéticos para evitar o crescimento de microrganismos, o fungo $P$. lilacinum se mantém viável nesses materiais e pode causar doenças que podem se complicar e causar morte (Khalique et al., 2018).

O tratamento de infecções causadas por $P$. lilacinum pode ser dificultado, pois o organismo apresenta resistência a vários dos principais antifúngicos utilizados na terapia atualmente, os "azóis" (Pastor e Guarro, 2006, Aguilar et al., 1998). Observam-se casos em que os testes de susceptibilidade apontam sensibilidade do fungo a uma dada molécula, mas a terapêutica não funciona como esperado (Hall et al., 2004). Os estudos dos mecanismos que promovem resistência têm se intensificado e se fazem muito importantes, dado o aumento de relatos de organismos resistentes a essas moléculas, como é o caso do $P$. lilacinum.

\section{Objetivos gerais}

O presente trabalho teve como objetivo geral explorar molecularmente o fungo $P$. lilacinum com relação à sua capacidade de resistir a antifúngicos e à sua capacidade de produzir peptidases robustas, com potencial de aplicação biotecnológica.

Como objetivos específicos, separamos em dois capítulos, para que a linha de raciocínio faça mais sentido.

Capítulo 1: resistência a antifúngicos

- Cultivar $P$. lilacinum em condições capazes de estimular a resistência aos antifúngicos fluconazol e itraconazol 
uma flexibilização dessa região por quebra de ligações fracas. $O$ efeito dessa flexibilização seria uma ativação mais eficiente da enzima, o que teria gerado maiores atividades nessa condição.

O fato da estrutura secundária da $r P I$ SerPep ter uma alta concentração de $\alpha$-hélices e o envelope molecular mostrar uma proteína globular coêsa provavelmente influenciam na sua estabilidade térmica. Estudos apontam que a presença de $\alpha$-hélices estáveis podem trazer termoestabilidade para as enzimas. Além disso, a coesão da molécula dificulta a desnaturação térmica, provocada por aumento de energia cinética da solução.

Os resultados da rPI_MetPep ainda serão complementados para que se tenha uma visão geral de sua atividade perante os substratos testados.

\section{Conclusão geral}

$\mathrm{O}$ presente trabalho mostrou diferentes abordagens moleculares que ajudaram a melhor descrever o fungo $P$. lilacinum. Este fungo apresenta importância agropecuária $e$ médica e sua completa descrição é de interesse pois, apesar de ser um patógeno emergente, possui um perfil genético com grande potencial biotecnológico.

Os conhecimentos gerados foram que o fungo lança mão de controle de expressão de genes específicos para conseguir se tornar resistente a antifúngicos e que possui em seu genoma genes de peptidases com potencial de aplicação industrial. Principalmente uma serino peptidase termotolerante.

\section{Referências bibliográficas}

Aguilar, C., Pujol, I., Sala, J., Guarro, J. Antifungal susceptibilities of Paecilomyces species. Antimicrobial Agents and Chemotherapy, 42(7), 1601-4. (1998)

Albesiano, E., Messmer, B. T., Damle, R. N., Allen, S. L., Rai, K. R., Chiorazzi, N. Activation-induced cytidine deaminase in chronic lymphocytic leukemia $B$ cells: expression as multiple forms in a dynamic, variably sized fraction of the clone. Blood. 102(9), 3333-9. (2003). 
Alcazar-Fuoli L, Mellado E. Ergosterol biosynthesis in Aspergillus fumigatus: its relevance as an antifungal target and role in antifungal drug resistance. Frontiers in Microbiology. 2012;3:439. doi:10.3389/fmicb.2012.00439.

Altschul SF; Gish W; Miller W; Myers EW; Lipman DJ., Basic local alignment search tool. J Mol Biol 215: 403-10 (1990).

Andrews, S. FastQC A Quality Control tool for High Throughput SequenceData. Version 0.11.8. Babraham Institute. http://www.bioinformatics.babraham.ac.uk/projects/fastqc/. (2018).

Banerjee B, Greenberger PA, Fink JN, Kurup VP. Immunological Characterization of Asp f 2, a Major Allergen from Aspergillus fumigatus Associated with Allergic Bronchopulmonary Aspergillosis. Kozel TR, ed. Infection and Immunity. 1998;66(11):5175-5182.

Banu, J. G., Rohini, I., and Gunasekaran, M. Mass multiplication and formulation of a nematophagous fungus, Paecilomyces lilacinus. Int. J. Nematol. 16, 145-152. (2006).

Blosser SJ, Cramer RA. SREBP-dependent triazole susceptibility in Aspergillus fumigatus is mediated through direct transcriptional regulation of erg11A (cyp51A). Antimicrob Agents Chemother 2012; 56:248-257. http://dx.doi.org/10.1128/AAC.05027-11.

Boettner, M.; Prinz, B.; Holz, C.; Stahl, U.; Lang, C. (2002) High-throughput screening for expression of heterologous proteins in the yeast Pichia pastoris. Journal of Biotechnology. v. 99, May. p. 51-62.

Bradford, M. M. A rapid and sensitive method for the quantitation of microgram quantities of protein utilizing the principle of protein-dye binding. Anal Biochem. 72, 248-54 (1976).

Burhan, Abbas $\mathrm{H}$; Annon, Mohammed R., Pathogenesis of Paecilomyces lilacinus against the immature stages of Musca domestica L. Journal of Pharmaceutical Sciences and Research; Cuddalore Vol. 11, Ed. 4 : 1595-1601. (2019).

Burmeister Getz E., Xiao M., Chakrabarty T., Cooke R., Selvin P.R. A comparison between the sulfhydryl reductants tris(2-carboxyethyl)phosphine and dithiothreitol for use in protein biochemistry. Anal. Biochem.,273:73-80. doi: 10.1006/abio.1999.4203. (1999).

Carey, J., D'Amico, R., Sutton, D. A., \& Rinaldi, M. G. Paecilomyces lilacinus vaginitis in an immunocompetent patient. Emerging Infectious Diseases, 9(9), 1155-8. (2003). 
Carmona, A. K.; Juliano, M. A.; Juliano, L. The use of Fluorescence Resonance Energy Transfer (FRET) peptides for measurement of clinically important proteolytic enzymes. Anais da Academia Brasileira de Ciências, v. 81, n. 3, p. 381-92, 2009.

Castellani, A. Maintenance and cultivation of the common pathogenic fungi of man in sterile distilled water. Further researches. J. trop Med. Hyg., 70, 181-184. (1967).

Chalissery, J., Jalal, D., Al-Natour, Z., Hassan, A. H. Repair of oxidative DNA damage in Saccharomyces cerevisiae. DNA Repair (Amst). 51, 2-13. doi: 10.1016/j.dnarep.2016.12.010. (2017).

Chamilos, G., Kontoyiannis, D. P. Update on antifungal drug resistance mechanisms of Aspergillus fumigatus. Drug Resist. Updat. 8 344-358. 10.1016/j.drup.2006.01.001. (2005).

Chowdhary, A., Kathuria, S., Xu, J., Meis, J, F. Emergence of azole-resistant Aspergillus fumigatus strains due to agricultural azole use creates an increasing threat to human health. PLoS Pathog. 9(10), e1003633. (2013).

Como JA, Dismukes WE. 1994. Oral azole drugs as systemic antifungal therapy. N Engl J Med 330:263-272. http://dx.doi.org/10.1056 /NEJM199401273300407.

DelMar E, Largman C, Brodrick J, Geokas M., A sensitive new substrate for chymotrypsin. Anal Biochem. 99:316-320. doi:10.1016/s0003-2697(79)80013-5. (1979).

Dias BA, Neves PMOJ, Furlaneto-Maia L, Furlaneto MC. Cuticle-degrading proteases produced by the entomopathogenic fungus Beauveria bassiana in the presence of coffee berry borer cuticle. Brazilian Journal of Microbiology. 2008;39(2):301-306. doi:10.1590/S1517-838220080002000019.

Dobin, A., et al. STAR: ultrafast universal RNA-seq aligner. Bioinformatics(Oxford, England), 29(1), 1521. (2012).

El-Gendy, M. M. A. (2010). Keratinase production by endophytic Penicillium spp. Morsy1 under solidstate fermentation using rice straw. Appl. Biochem. Biotechnol. 162, 780-794. doi: 10.1007/s12010009-8802-x

Espadale, E., Buckley, L., Borio, S., McEwan, N., Schmidt, V. Successful multimodal treatment of Paecilomyces lilacinus infection in a dog Veterinary Record Case Reports 6: e000627. doi: 10.1136/vetreccr-2018-000627. (2018) 
Evans, J. M., Wang, A. L., \& Elewski, B. E. Successful treatment of Paecilomyces lilacinus onychomycosis with efinaconazole and tavaborole. Skin Appendage Disorders. 1(4), 169-71 (2016).

Finn R.D., Clements J., Eddy S.R. HMMER web server: interactive sequence similarity searching., Nucleic Acids Research (2011) Web Server Issue 39:W29-W37.

Fischer, $\mathrm{H}$. et al. Determination of the molecular weight of proteins in solution from a single small-angle X-ray scattering measurement on a relative scale. Journal of Applied Crystallography, v. 43, n. 1, p. 101-109, 2009.

Fonseca, L. C., Corrêa, N. C. R., Garrote-Filho, M. da S., Cunha, C. C. da, e Penha-Silva, N., Efeito da composição do solvente sobre a estabilidade de proteínas em soluções aquosas. Química Nova, 29(3), 543-548. https://dx.doi.org/10.1590/S0100-40422006000300024. (2006).

Giosa D, Felice MR, Lawrence TJ, et al. Whole RNA-Sequencing and Transcriptome Assembly of Candida albicans and Candida africana under Chlamydospore-Inducing Conditions. Genome Biology and Evolution. 2017;9(7):1971-1977. doi:10.1093/gbe/evx143.

Giovanini G. T., Produção, liofilização, purificação e determinação de especificidade da peptidase isolada do fungo Scopulariopsis koningii. Dissertação de mestrado apresentada ao Programa de PósGraduação em Ciências Farmacêuticas da USP - Ribeirão Preto para obtenção do Título de Mestre em Ciências. Área de Concentração: Produtos Naturais e Sintéticos., Orientador: Prof. Dr. Hamilton Cabral. 2014.

Graninger W., Diab-Elschahawi M., Presterl E. (2019) Antifungal Agents. In: Presterl E. (eds) Clinically Relevant Mycoses. Springer, Cham

Gupta R., E. Jung and S. Brunak. Prediction of N-glycosylation sites in human proteins. In preparation, 2004.

Guzman, M. L., Marques, M. R., Olivera Me, M. E., \& Stippler, E. S., Enzymatic activity in the presence of surfactants commonly used in dissolution media, Part 1: Pepsin. Results in pharma sciences, 6, 1519. doi:10.1016/j.rinphs.2016.02.002. (2016).

Haas BJ, Papanicolaou A, Yassour M, Grabherr M, Blood PD, Bowden J, Couger MB, Eccles D, Li B, Lieber M, Macmanes MD, Ott M, Orvis J, Pochet N, Strozzi F, Weeks N, Westerman R, William T, Dewey CN, Henschel R, Leduc RD, Friedman N, Regev A. De novo transcript sequence reconstruction from RNA-seq using the Trinity platform for reference generation and analysis. Nat Protoc. 2013 
Aug;8(8):1494-512. Open Access in PMC doi: 10.1038/nprot.2013.084. Epub 2013 Jul 11. PubMed PMID:23845962.

Hall T. A., BioEdit: a user-friendly biological sequence alignment editor and analysis program for Windows 95/98/NT. Nucl. Acids. Symp. Ser. 41: 95-98. (1999).

Hall, V. C., Goyal, S., Davis, M. D. P., Walsh, J.S. Cutaneous hyalohyphomycosis caused by Paecilomyces lilacinus: report of three cases and review of the literature. Int J Dermatol, 43, 648-653. (2004)

Hamin Neto, Y. A. A.; De Oliveira, L. C. G., De Oliveira, J. R. ; Juliano, M. A. ; Juliano, L., Arantes, E. C. ; Cabral, H., Analysis of the Specificity and Biochemical Characterization of Metalloproteases Isolated from Eupenicillium javanicum Using Fluorescence Resonance Energy Transfer Peptides. Frontiers in Microbiology (Online), v. 7, p. 1., (2017).

Hatanaka, T., Arima, J., Uesugi, Y., and Iwabuchi, M. (2005). Purification, characterization cloning, and sequencing of metalloendopeptidase from Streptomyces septatus TH-2. Arch. Bioquem. Biophys. 434, 289-298. doi: 10.1016/j.abb.2004.11.018

Hokken, M., et al. Phenotypic plasticity and the evolution of azole resistance in Aspergillus fumigatus; an expression profile of clinical isolates upon exposure to itraconazole. BMC genomics, 20(1), 28. doi:10.1186/s12864-018-5255-z. (2019).

Illumina. TruSeq RNA Sample Preparation kit v2. Illumina website.https://support.illumina.com/content/dam/illumina-

support/documents/documentation/chemistry_documentation/samplepreps_truseq/truseqrna/truseqrna-sample-prep-v2-guide-15026495-f.pdf. (2014).

Jatala P, Kaltenbach R, Bocangel M. "Biological control of Meloidogyne incognita acrita and Globodera pallida on potatoes". Journal of Nematology. 11: 303. (1979).

Kelley, L. A., Mezulis, S.Yates, C. M., Wass, M. N., Sternberg, M. J. E. The Phyre2 web portal for protein modeling, prediction and analysis., Nature Protocols., 10:845 DOI: 10.1038/nprot.2015.053 (2015)

Khalique Z, Hatipoğlu S, Rosendahl U, Mohiaddin R. Unusual complicated fungal endocarditis in a patient with vascular Ehlers-Danlos Syndrome. The Annals of Thoracic Surgery. doi: https://doi.org/10.1016/j.athoracsur.2018.08.074_(2018) 
Khan A, Williams KL, Nevalainen HK (2004). "Effects of Paecilomyces lilacinus protease and chitinase on the eggshell structures and hatching of Meloidogyne javanica juveniles". Biological Control. 31 (3): 346-52. doi:10.1016/j.biocontrol.2004.07.011

Khan, Z., et al.Purpureocillium lilacinum as a cause of cavitary pulmonary disease: a new clinical presentation and observations on atypical morphologic characteristics of the isolate. Journal of Clinical Microbiology. 50(5), 1800-4 (2012).

Kiewnick, S., Neumann, S., Sikora, R. a., and Frey, J. E. (2011). Effect of Meloidogyne incognita inoculum density and application rate of Paecilomyces lilacinus strain 251 on biocontrol efficacy and colonization of egg masses analyzed by real-time quantitative PCR. Phytopathology 101, 105-112. doi: 10.1094/PHYTO-03-10-0090

Kontoyiannis, D.P., Sagar, N., Hirschi, K.D. Ovrexpression of Erg11p by the regulatable GAL1 promoter confers fluconazole resistance in Saccharomyces cerevisiae. Antimicrob. Agents Chemother. 43, 2798-2800. (1999).

KOZIN, M. B.; SVERGUN, D. I. Automated matching of high- and low-resolution structural models. Journal of Applied Crystallography, v. 34, n. 1, p. 33-41, Feb. 2001.

Krogh A, Larsson B, von Heijne G, Sonnhammer EL. Predicting transmembrane protein topology with a hidden Markov model: application to complete genomes. J Mol Biol. 2001 Jan 19;305(3):567-80.

Leonardelli F, Macedo D, Dudiuk C, Cabeza MS, Gamarra S, Garcia-Effron G. Aspergillus fumigatus Intrinsic Fluconazole Resistance Is Due to the Naturally Occurring T301I Substitution in Cyp51Ap. Antimicrobial Agents and Chemotherapy. 2016;60(9):5420-5426. doi:10.1128/AAC.00905-16.

Li, B., \&amp; Dewey, C. N. RSEM: accurate transcript quantification from RNA-Seq data with or without a reference genome. BMC Bioinformatics, 12, 323.doi:10.1186/1471-2105-12-323. (2011).

Li, W., and Godzik A. , CD-HIT: a fast program for clustering and comparing large sets of protein or nucleotide sequences. Bioinformatics 22:1658-1659. (2006).

Lopes N. P., Pesquisas de novas enzimas levam Nobel de Química 2018., 2018. Acessado em 12/06/2019 em https://jornal.usp.br/artigos/pesquisas-de-novas-enzimas-levam-nobel-de-quimica2018/ 
Love M. I., Huber W., Anders S. Moderated estimation of fold change anddispersion for RNA-seq data with DESeq2. Genome Biology, 15, 550. doi:10.1186/s13059-014-0550-8. (2014).

Luangsa-ard J., et al. Purpureocillium, a new genus for the medically important Paecilomyces lilacinus. FEMS Microbiol. Lett. 321, 141-149. (2011).

Manente, M., Ghislain, M. The lipid-translocating exporter family and membrane phospholipid homeostasis in yeast. FEMS Yeast Res. 9(5), 673-687. doi: 10.1111/j.1567-1364.2009.00513.x. (2009).

Mansfield BE, Oltean HN, Oliver BG, Hoot SJ, Leyde SE, Hedstrom L, White TC. 2010. Azole drugs are imported by facilitated diffusion in Candida albicans and other pathogenic fungi. PLoS Pathog 6:e1001126. http://dx.doi.org/10.1371/journal.ppat.1001126.

Merheb-Dini, C., Cabral, H., Leite, R. S. R., Zanphorlin, L. M., Okamoto, D. N., Bonilla Rodriguez, G. O., et al. (2009). Biochemical and functional characterization of ametalloprotease from the thermophilic fungus Thermoascus aurantiacus. J. Agric. Food Chem. 57, 9210-9217. doi: 10.1021/jf9017977

Mordor Intelligence., Industrial enzymes market - growth, trends, and forecast (2019 - 2024)., 2018. Acessado em 13/06/2019 em https://www.mordorintelligence.com/industry-reports/industrialenzymes-market

NCCLS. Reference method for broth dilution antifungal susceptibility testing of filamentous fungi. Approved Standard. NCCLS document M38-A [ISBN 1- 56238-470-8]. 19087-1898 USA. (2002).

Pastor FJ, Guarro J. Clinical manifestations, treatment and outcome of Paecilomyces lilacinus infections. Clin Microbiol Infect. 12, 948-60 (2006).

Pedezzi, R., $\beta$-frutofuranosidases em coleópteros: caracterização funcional e produção heteróloga de uma $\beta$-frutofuranosidase de Sphenophorus levis. Dissertação de mestrado apresentada ao Programa de Pós-Graduação em Genética Evolutiva e Biologia Molecular da Universidade Federal de São Carlos. Orientador: Flavio Henrique Silva (2015)

Petersen T. N., Søren Brunak, Gunnar von Heijne \& Henrik Nielsen. SignalP 4.0: discriminating signal peptides from transmembrane regions. Nature Methods, 8:785-786, 2011 
Pradhan, A., et al. Elevated catalase expression in a fungal pathogen is a double-edged sword of iron. PLoS pathogens, 13(5), e1006405. doi:10.1371/journal.ppat.1006405. (2017).

Prasad, P., Varshney, D. \& Adholeya, A. Whole genome annotation and comparative genomic analyses of bio-control fungus Purpureocillium lilacinum. BMC Genomics. 16, 1004. doi:10.1186/s12864-015-2229-2 (2015).

Prasad, R., Rawal, M. K. Efflux pump proteins in antifungal resistance. Frontiers in Pharmacology, 5, 202. doi:10.3389/fphar.2014.00202. (2014).

Raghavan, R., Chithra, G., Fernandez, S., Shamana Suryanarayana, B., \& Singh, R. An unusual case of hyalohyphomycosis due to Purpureocillium lilacinum in a patient with myasthenia gravis. Current Medical Mycology. 4(2), 36-39 (2018).

Sanadhya, P., Bucki, P., Liarzi, O., Ezra, D., Gamliel, A., \& Braun Miyara, S. Caenorhabditis elegans susceptibility to Daldinia cf. concentrica bioactive volatiles is coupled with expression activation of the stress-response transcription factor daf-16, a part of distinct nematicidal action. PloS one, 13(5), e0196870. doi:10.1371/journal.pone.0196870. (2018).

Sanglard, D., Ischer, F., Calabrese, D., de Micheli, M., Bille, J. Multiple resistance mechanisms to azole antifungals in yeast clinical isolates. Drug Resist. Updat. 1, 255-265. (1998).

Sarath, G., De La Motte, R. S., Wagner, F. W., Peptidase assay methods. In: Beynon, R.J., BOND, J.S. Proteolytic enzymes a practical approach. Oxford University, New York, cap. 3, p. 25-55. 1996.

Scorzoni, L., et al. Antifungal therapy: new advances in the understanding and treatment of mycosis. Frontiers in Microbiology, 8, 36. doi:10.3389/fmicb.2017.00036. (2017).

Sharma S., Vaid S., Bhat B., Singh S., Bajaj B. K., Chapter 17 - Thermostable Enzymes for Industrial Biotechnology., Editor(s): Ram Sarup Singh, Reeta Rani Singhania, Ashok Pandey, Christian Larroche., In Biomass, Biofuels, Biochemicals.,Advances in Enzyme Technology, Elsevier., 2019, Pages 469-495., ISBN 9780444641144., acessado em 11/06/2019. https://doi.org/10.1016/B978-0444-64114-4.00017-0. (http://www.sciencedirect.com/science/article/pii/B9780444641144000170) Shivaprasad, A., Ravi, G. C., Shivapriya, \& Rama. A Rare case of nasal septal perforation due to Purpureocillium lilacinum: case report and review. Indian Journal of Otolaryngology and Head and Neck Surgery : official publication of the Association of Otolaryngologists of India. 65(2), 184-8 (2012). 
Srinivasan, A., Lopez-Ribot, J. L., \& Ramasubramanian, A. K. Overcoming antifungal resistance. Drug discovery today. Technologies, 11, 65-71. (2014).

Statista., Biotechnology industry - Statistics \& Facts., 2018. Acessado em 13/06/2019 em https://www.statista.com/topics/1634/biotechnology-industry/

Steentoft C, Vakhrushev SY, Joshi HJ, Kong Y, Vester-Christensen MB, Schjoldager KT, Lavrsen K, Dabelsteen S, Pedersen NB, Marcos-Silva L, Gupta R, Bennett EP, Mandel U, Brunak S, Wandall HH, Levery SB, Clausen H. Precision mapping of the human O-GalNAc glycoproteome through SimpleCell technology. EMBO J, 32(10):1478-88, May 15, 2013. (doi: 10.1038/emboj.2013.79. Epub 2013).

Stirling GR, West LM. "Fungal parasites of root-knot nematode eggs from tropical and sub-tropical regions of Australia". Australasian Plant Pathology. 20 (4): 149-54. doi:10.1071/APP9910149. (1991).

Sun, M., and Liu, X. Carbon requirements of some nematophagous, entomopathogenic and mycoparasitic Hyphomycetes as fungal biocontrol agents. Mycopathologia 161, 295-305. doi: 10.1007/s11046-006-0249-9. (2006).

Svergun, D. I. Determination of the regularization parameter in indirect-transform methods using perceptual criteria. Journal of Applied Crystallography, v. 25, n. 4, p. 495-503, 1992.

Tangrea M. A., Bryan P. N., Sari N., Orban J., Solution Structure of the Pro-hormone Convertase 1 Pro-domain from Mus musculus., Journal of Molecular Biology., Volume 320, Issue 4, Pages 801-812, ISSN 0022-2836, https://doi.org/10.1016/S0022-2836(02)00543-0. (2002)

Thys, R. C. S., and Brandelli, A. (2006). Purification and properties of a keratinolytic metalloprotease from Microbacterium sp. J. Appl. Microbiol. 101, 1259-1268. doi: 10.1111/j.1365-2672.2006.03050.x

Trinh, S. A., \& Angarone, M. P. Purpureocillium lilacinum tattoo-related skin infection in a kidney transplant recipient. Transplant Infectious Disease: an Official Journal of the Transplantation Society, 19(3). 10.1111/tid.12689 (2017).

Vandeputte P, Ferrari S, Coste AT. 2012 Antifungal resistance and new strategies to control fungal infections. Int J Microbiol 2012:713687. http: //dx.doi.org/10.1155/2012/713687. 
Visser H., Joosten V., Punt P. J., Gusakov A. V., Olson P. T., Joosten R., Bartels J., Visser J., Sinitsyn A. P., Emalfarb M. A., Verdoes J. C., Wery J., Development of a mature fungal technology and production platform for industrial enzymes based on a Myceliophthora thermophila isolate, previously known as Chrysosporium lucknowense C1., Industrial Biotechnology. Jun 2011.acessado em 12/06/2019 em http://doi.org/10.1089/ind.2011.7.214

VOLKOV, V. V.; SVERGUN, D. I. Uniqueness of ab initio shape determination in small-angle scattering. Journal of Applied Crystallography, v. 36, n. 3, p. 860-864, 2003.

Walsh, TJ, Groll, A, Hiemenz, J, Fleming, R, Roilides, E, and Anaissie, E. Infections due to emerging and uncommon medically important fungal pathogens. Clin Microbiol Infect. 2004; 10: 48-66

Wang, G., et al. Biosynthesis of antibiotic leucinostatins in bio-control fungus Purpureocillium lilacinum and their inhibition on phytophthora revealed by genome mining. PLoS Pathogens, 12(7), e1005685. doi:10.1371/journal.ppat.1005685 (2016).

Wang, J., Wang, J., Liu, F., and Pan, C. Enhancing the virulence of Paecilomyces lilacinus against Meloidogyne incognita eggs by overexpression of a serine protease. Biotechnol. Lett. 32, 1159-1166. doi: 10.1007/s10529-010-0278-9. (2010).

Xie, J., et al. Genome and transcriptome sequences reveal the specific parasitism of the nematophagous Purpureocillium lilacinum 36-1. Frontiers in microbiology. 7, 1084. doi:10.3389/fmicb.2016.01084 (2016).

Yakimov, A. P., Afanaseva, A. S., Khodorkovskiy, M. A., \& Petukhov, M. G. (2016). Design of Stable a-Helical Peptides and Thermostable Proteins in Biotechnology and Biomedicine. Acta naturae, 8(4), 70-81.

Yang L., Peng M., Shah S. S., Wang Q., Transcriptome Sequencing and Comparative Analysis of Piptoporus betulinus in Response to Birch Sawdust Induction. Forests 2017, 8, 374; doi:10.3390/f8100374.

Yue, Y., et al. Exploring the antibacterial and antifungal potential of jellyfish-associated marine fungi by cultivation-dependent approaches. PloS One, 10(12), e0144394. doi:10.1371/journal.pone.0144394 (2015).

Zibaee A., Ramzi S., Cuticle-degrading proteases of entomopathogenic fungi: from biochemistry to biological performance., Archives of Phytopathology and Plant Protection., 51 13-14 779-794 Doi: https://doi.org/10.1080/03235408.2018.1519875 (2018) 